\title{
Os mecanismos de socialização e a criação de valor no relacionamento cliente-fornecedor
}

\section{Socialization mechanisms and value creation in buyer-supplier relationships}

\author{
Cristiane Biazzin Villar ${ }^{1}$ \\ Susana Carla Farias Pereira ${ }^{1}$
}

\begin{abstract}
Resumo: Este artigo tem por objetivo investigar o impacto dos mecanismos de socialização na criação de valor no relacionamento cliente-fornecedor. Desenvolvido a partir da visão relacional e dos mecanismos de socialização, argumenta-se que o relacionamento cliente-fornecedor pode impactar positivamente a cadeia de suprimentos. Foi adotado o método de pesquisa Survey, desenvolvida com 91 executivos de Compras e Supply Chain no Brasil, e a amostra foi analisada utilizando o modelo de equações estruturais. A principal contribuição desta pesquisa é a presença de baixas evidências empíricas, indicando que o capital relacional está associado a altos níveis de ganhos.

Palavras-chave: Supply chain management. Mecanismos de socialização. Capital relacional. Modelo de equações estruturais. Relacionamento comprador-fornecedor.
\end{abstract}

\begin{abstract}
This paper aims to investigate the impact of socialization mechanisms for value creation in buyer-supplier relationships. Drawing upon relational view and socialization mechanisms, it is argued that the buyer-supplier relationship can provide positive outcomes for its supply chain. Structural Equation Modeling performed on data collected from Purchasing and Supply Chain Executives indicates that both formal and informal socialization mechanisms positively impact the level of relational capital of supply chain. The main contribution presented by this research is the non-strong empirical evidence, indicating that relational capital is associated with higher levels of earnings for firms.
\end{abstract}

Keywords: Supply chain management. Socialization mechanisms. Relational capital. Structural equation modeling. Buyer-supplier relationship.

\section{Introdução}

As primeiras discussões sobre o processo de socialização dentro das organizações iniciaram-se nos estudos de comportamento organizacional. Até o final dos anos 1970, os estudos sobre socialização eram focados no indivíduo e em sua participação em grupos de uma forma geral, na qual este poderia aprender por meio da interação com os demais e entender quem desenvolvia as regras sobre o que. A tratativa dada até então se limitava ao reconhecimento ou punição de ações corretas e incorretas (LOUIS, 1980).

A partir do final dos anos 1970, a socialização organizacional redirecionou seu foco do indivíduo para uma ótica coletivista e intraorganizacional. Este movimento pode ser percebido nos estudos de Van Maanen (1978) e Louis (1980). A socialização organizacional passa, então, a ser vista como o processo pelo qual um indivíduo vem a apreciar valores organizacionais, desenvolver habilidades, comportamentos esperados e o conhecimento social essencial para assumir uma responsabilidade ou

função organizacional para, assim, participar como um membro de determinada instituição. Novos entrantes deveriam aprender as regras e culturas de dentro e de fora da própria organização (LOUIS, 1980). De modo geral, a socialização organizacional era considerada como o processo pelo qual um indivíduo adquire o conhecimento social e as competências necessárias para assumir seu papel na organização (VAN MAANEN, 1978).

Nesta fase, também, economistas e sociólogos passaram a investigar os fluxos de informação como sendo processos de formação de redes para compreender seu significado para o âmbito corporativo (MARTES et al., 2006). Até então, evidências empíricas sugerem que o número de estudos sobre socialização interorganizacionais era baixo, porém, a partir do final dos anos 1980, houve um aumento de interesse significativo nos relacionamentos interorganizacionais (GULATI; GARGIULO, 1999) e o interesse sobre este tema ainda permanece (TERPEND et al., 2008;

\footnotetext{
${ }^{1}$ Departamento de Produção e Operações, Escola de Administração de Empresas de São Paulo, Fundação Getúlio Vargas - FGV, Rua Itapeva, 474, 8o. andar, CEP 01313-902, São Paulo, SP, Brasil, e-mail: cristiane.villar@fgv.br; Susana.pereira@fgv.br
} 
KIM et al., 2010; LUMINEAU; HENDERSON, 2012; SCHOENHERR et al., 2012). O rápido crescimento deste interesse de pesquisa deve-se ao fato de o estabelecimento de alianças e parcerias ter sido reconhecido cada vez mais como uma forma mais institucionalizada de cooperação entre empresas (GULATI, 1998) e da percepção de que as empresas que possuem o expertise de gerenciar seus relacionamentos interorganizacionais podem obter melhores condições para atingir maiores níveis de vantagem competitiva. Em outras palavras, essas empresas poderiam se beneficiar do capital relacional criado a partir dessas relações (DYER; SINGH, 1998).

Este tema é alvo de discussão na literatura. Enquanto diversos autores reforçam os aspectos relacionais e de socialização como positivos para a criação de valor (DYER; SINGH, 1998; CARR; PEARSON, 1999; ANDERSEN; BUVIK, 2001; HANDFIELD; BECHTEL, 2001; COUSINS et al., 2006; LAWSON et al., 2009, CAREY et al., 2011; STOUTHUYSEN; SLABBINCK; ROODHOOFT, 2012), outros autores discordam e argumentam que, por vezes, estes aspectos podem dificultá-la (COX, 2004; DYER; HATCH, 2004; VILLENA; REVILLA; CHOI, 2011). Esta discussão conduz este estudo a explorar as seguintes perguntas de pesquisa: $O s$ mecanismos de socialização impactam positivamente a geração de capital relacional? Este impacto é percebido como direcionador de resultados superiores no relacionamento com fornecedores?

A presente pesquisa objetiva verificar empiricamente a existência de evidências que demonstrem o impacto dos mecanismos formais e informais de socialização na geração de capital relacional e, consequentemente, na percepção de desempenho operacional da firma. Para atender ao objetivo geral da pesquisa, o presente artigo está estruturado da seguinte forma: inicialmente avaliar-se-á teoricamente o processo de formação do relacionamento interorganizacional, bem como a conceituação e aplicabilidade dos mecanismos de socialização e o conceito de capital relacional. Esta primeira etapa objetiva compreender a conexão entre socialização e capital relacional, com a revisão da literatura disponível, buscando o estado da arte em que o tema se encontra. Em seguida, apresenta-se a metodologia de estudo adotada, a caracterização dos construtos, os procedimentos de pré-teste e o detalhamento da amostra utilizada. Explora-se a análise dos dados coletados, o modelo de equações estruturais e os procedimentos de ajuste do modelo. Finaliza-se o presente artigo com as conclusões, limitações e oportunidades de pesquisa futura.

\section{O Relacionamento interorganizacional}

Este estudo parte do pressuposto de que a firma não opera de forma isolada das demais (SKJOETTLARSEN, 1999). Ao contrário, todas as ações e considerações organizacionais podem ser realmente percebidas por aqueles que a compõem e seus relacionamentos. Neste contexto, reside o argumento em que os comportamentos e as instituições a serem analisados são tão compelidos pelas constantes relações sociais que seria um mal entendido considerável a sua interpretação como elemento isolado (GRANOVETTER, 2007).

A interpretação que Granovetter (2007) elabora sobre o tema leva em conta que a maior parte do comportamento está profundamente imersa em redes de relações interpessoais e que essa abordagem evita os extremos das visões sub e supersocializada da ação humana. Este autor busca conceituar a imersão sob uma vertente mais humanizada, destacando que os atores buscam agir com propósito, cujas ações estariam imersas em sistemas concretos e contínuos de relações sociais, ao invés de tomar ações e/ou comportamentos fora de um contexto social ou adotar um roteiro escrito e pré-definido para eles pela intersecção específica de categorias sociais que porventura ocupem. $\mathrm{O}$ autor utiliza como arcabouço à sua fundamentação teórica, entre outros pontos, a questão da "má-fé vs. confiança", em que os relacionamentos interorganizacionais estão dinamicamente inseridos.

Os termos relações e relacionamento são utilizados na academia para discussão de práticas de negócios. Isso retrata também o nível de preocupação de acadêmicos e profissionais em compreender como as firmas se relacionam, e conhecer os problemas e oportunidades encontradas (OLIVER, 1990; VASCONCELOS; MILAGRES; NASCIMENTO, 2005). A palavra "relacionamento" indica a existência de alguma forma organizacional diferente, um nível superior ao da rotina das empresas de forma individual. As organizações entram em determinadas relações de forma consciente e por razões específicas e com uma variedade de condições que possam limitar ou influenciar suas escolhas (OLIVER, 1990). O relacionamento interorganizacional pode ser compreendido como um processo em que há cooperação voluntária, à qual as organizações combinam recursos para superar a incerteza criada por forças do ambiente que, por ventura, estejam além do seu controle (GULATI; GARGIULO, 1999).

As organizações estabelecem relações ou mecanismos de troca com outras organizações por uma questão, inicialmente, de necessidade (OLIVER, 1990; GULATI, 1998) e de forma voluntária (GULATI; GARGIULO, 1999). Na verdade, por iniciativa própria, uma firma identifica a necessidade de estabelecer um relacionamento ou até uma aliança, busca o melhor parceiro disponível e escolhe o melhor instrumento para formalizar essa união, seja formal ou informal (GULATI, 1998).

As firmas, de maneira geral, não possuem todos os recursos para atender à demanda de seus clientes. As 
firmas são dependentes de conhecimento, competências, recursos, ações e intenções de fornecedores, distribuidores, outros clientes e, por vezes, até de competidores para atender a todos os requisitos. Nesse sentido, o capital relacional é um recurso acessível e disponível a um grupo seleto de atores construído a partir do relacionamento estabelecido por estes (BOURDIER, 1980; COLEMAN, 1988). Por exemplo, o estudo de Wang, Tai e Varun (2013) enfatiza que, ao utilizar soluções tecnológicas de relacionamento, as organizações induzem a geração de respostas oriundas deste relacionamento (o que pode representar investimentos no processo dos fornecedores e avanços na flexibilidade), o que, consequentemente, traz resultados positivos a compradores e vendedores. A interdependência entre empresas significa que o processo estratégico é interativo, evolucionário e responsivo (OLIVER, 1990). Nesse processo interativo, novos laços podem ser estabelecidos de forma mais dinâmica (GULATI; GARGIULO, 1999).

Gulati (1998) sugere que os relacionamentos sociais não apenas influenciam a criação de novos laços como também afetam seu desenho, evolução e até seu sucesso. Oliver (1990) destaca como motivadores à formação de rede interorganizacionais, seis fatores contingenciais (necessidade, assimetria, reciprocidade, eficiência, estabilidade, legitimidade) e que, embora cada um possa possuir causas suficientemente distintas para a formação desses relacionamentos, essas contingências geralmente ocorrem simultaneamente.

O processo de formação de um relacionamento é constituído pela dedicação de tempo e dinheiro de seus participantes, aliados a expectativas de resultados futuros. Isso significa que uma empresa que esteja buscando uma mudança é dependente da aprovação e ações de outros para atingir tal mudança, seja a mudança de um processo logístico até o lançamento de um novo produto (HAKANSSON; FORD, 2002). Os autores destacam, entretanto, que uma empresa pode conduzir parte de sua rede para a direção que deseja, se suas ações forem desenhadas apropriadamente e se isto for visto pelas demais como algo positivo. Geralmente uma rede é o resultado de deliberações, expectativas e ações de certo número de participantes. Levando em conta que o objetivo geral desta pesquisa é verificar se os mecanismos de socialização interorganizacionais impactam a geração de capital relacional e a geração de valor, este estudo basear-se-á na dimensão interorganizacional.

\subsection{Implicações dos relacionamentos interorganizacionais para a gestão da cadeia de suprimentos}

A literatura sugere que as empresas que possuem o expertise de gerenciar seus relacionamentos interorganizacionais podem obter melhores condições para atingir vantagem competitiva (DYER; SINGH, 1998; FLYNN; HUO; ZHAO, 2010) e que os mecanismos de socialização podem favorecer esse desempenho superior (CARR; PEARSON, 1999; ANDERSEN; BUVIK, 2001; HANDFIELD; BECHTEL, 2001; COUSINS et al., 2006; LAWSON et al, 2009, CAREY et al., 2011; STOUTHUYSEN; SLABBINCK; ROODHOOFT, 2012).

Os estudos de Cousins e Menguc (2006) demonstram que, sob a perspectiva do fornecedor, é importante estabelecer canais claros de comunicação com os compradores. Isto faz com que a compreensão do nível de desempenho entre os atores seja demonstrada. $\mathrm{O}$ presente estudo ater-se-á à perspectiva da empresa compradora (focal) e, assim, o recorte delimitado restringir-se-á às medidas de desempenho operacional. Conforme Sacomano Neto e Pires (2012, p.743),

[...] pode-se constatar que as medidas a montante são fortemente direcionadas para os aspectos operacionais relacionados à qualidade, produção e desempenho das entregas.

Cousins e Menguc (2006) também sugerem que a integração da cadeia de suprimentos é um estágio do processo de socialização, o qual se inicia a partir da comunicação entre as partes, culminando na melhoria da performance operacional do fornecedor percebida pelo comprador, que, consequentemente, conduz a uma evolução no relacionamento interorganizacional. Complementarmente, Carr e Pearson (1999) afirmam que o relacionamento entre clientes e fornecedores tem um impacto positivo no desempenho da firma. Eles sugerem que os relacionamentos entre compradores e fornecedores têm se tornado mais cooperativo com fornecedores-chave, e é esperado que essas firmas tenham um nível maior de performance financeira. Seu estudo oferece evidências empíricas da importância em se manter e/ou dedicar atenção especial aos relacionamentos como forma de obtenção de vantagem competitiva, focando mais os relacionamentos a longo prazo ao invés de relacionamentos pontuais.

Carr e Pearson (1999) continuam sua tese, destacando que, em relacionamentos de longo termo, compradores e fornecedores têm a possibilidade de desenvolver relacionamentos que envolvam crescentes níveis de comunicação, cooperação e coordenação de todas as atividades associadas à produção de bens e serviços. Destacam ainda a possibilidade de, por conta dessa aproximação, ambos reduzirem custos. Os autores finalizam sua argumentação, enfatizando que, com base em seu estudo, pode-se perceber que firmas que utilizam relações adversariais (adversarial relationships) com fornecedores-chave podem experimentar o gosto amargo de desempenho financeiro e operacional inferior com o passar do tempo. Tal comportamento adverso com fornecedores 
pode render à empresa compradora certa desvantagem, principalmente quando o nível de dependência desta for significativo, tendo a possibilidade de ruptura do fornecimento de itens críticos e, eventualmente, impactar toda a cadeia de suprimentos.

Ao se observar a relação entre os mecanismos de socialização e desempenho superior, Campbell (1997), por exemplo, investigou se a qualidade e intensidade da cooperação em parcerias afetam positivamente os resultados e a performance de desenvolvimento de novos produtos. Os autores levaram em conta os níveis de resolução de problemas em conjunto, confiança, o envolvimento com a outra parte, o nível de delegação formal de responsabilidades, o comprometimento, o escopo do relacionamento e o nível de satisfação. Concluíram que apenas uma característica comportamental da parceria colaborativa afetaria o desempenho de desenvolvimento de novos produtos: o nível de envolvimento entre as partes.

Há evidências de que a responsividade é um dos melhores retornos que uma cadeia de suprimentos pode ter a partir do estabelecimento de relações interorganizacionais, com a redução do tempo de ciclo da cadeia de suprimentos (HANDFIELD; BECHTEL, 2001). Isto se dá por conta da pressão que as empresas têm sofrido para responder de forma mais rápida às mudanças das prioridades competitivas, diante de ambientes cada vez mais velozes e dinâmicos.

Dessa forma, a literatura sinaliza que os mecanismos formais e informais podem compor um conjunto de recursos relacionais (capital relacional) que, quando acessado, pode proporcionar um impacto significativo nos retornos e no desempenho dos fornecedores (COUSINS et al., 2006; HUGHES; PERROW, 2011; STOUTHUYSEN; SLABBINCK; ROODHOOFT, 2012).

\section{Conceituação e aplicabilidade dos mecanismos de socialização}

O termo mecanismo tem se tornado comum nas pesquisas em ciências sociais. (HEDSTRÖM; SWEDBERG, 1998). Para os autores, o mecanismo social é uma parte integral de uma explicação teórica, em que certa causa ou input gera um efeito ou resultado, esperado ou não. Em obra posterior, Hedström (2005, p. 11) trata os mecanismos sociais como "[...] uma constelação de entidades ou atividades que estão conectadas de forma a atingir um resultado específico". Assim, os mecanismos sociais serão considerados, no presente estudo, como o processo pelo qual a socialização organizacional pode ser operacionalizada e para acessar o capital relacional (resultado específico).

Os mecanismos de socialização focam a forma como a firma e seus integrantes interagem, e ajudam a compreender por que algumas firmas podem ter mais sucesso que outras em gerenciar seus relacionamentos na cadeia de suprimentos (COUSINS; LAWSON, 2006). No contexto do relacionamento clientefornecedor, os mecanismos de socialização possibilitam que cada ator desenvolva certos aprendizados no relacionamento interorganizacional com o intuito de criar certas normas sociais e compartilhar conhecimentos.

O estabelecimento de novos relacionamentos é um processo importante para que as organizações possam desenvolver confiança em seus negócios (HILL et al., 2009), adicionalmente ao sucesso ou experiência que cada empresa possua individualmente. Os mecanismos de socialização oferecem significativa contribuição nesse sentido, ao favorecer a construção da confiança e o respeito no relacionamento, assim como gerar instrumentos direcionadores aos atores durante eventos excepcionais, como conflitos, incertezas ou problemas de fornecimento (COUSINS; LAWSON, 2006; HILL et al., 2009). A socialização, nesse sentido, é compreendida como o nível de interação entre os diversos atores que favorece sua aproximação e familiaridade e, consequentemente, pode melhorar seu processo de comunicação e resolução de problemas (GUPTA; GOVINDARAJAN, 2000; LAWSON et al., 2009).

Os quatro princípios fundamentais para caracterização de um mecanismo (HEDSTRÖM; SWEDBERG, 1998) são:

- Ação - leva em conta que são os atores que agem, e não suas variáveis. Os mecanismos não são construídos por meras associações entre as variáveis e sim referem-se diretamente às causas e consequências de ações individuais orientadas ao comportamento de outras;

- Precisão - captura-se a essência sociológica e expressa-se a ideia de que os mecanismos devem ser precisos, e não podem ser prematuros, superficiais ou vagos;

- Abstração - expressa a ideia de que a teorização efetiva não é possível sem a eliminação imediata dos fatores irrelevantes e deve ser direcionada ao foco do assunto central;

- Redução - necessidade de se buscar um refinamento para a explicação do conceito.

A proposta de Hedström e Swedberg (1998) é: ao invés de analisar os relacionamentos exclusivamente no nível macro, destacar a necessidade de tentar estabelecer como os eventos no nível macro afetam o nível individual, como os indivíduos assimilam os impactos desses eventos no nível micro e como o número de indivíduos, por meio de suas ações e interações, geram resultados no nível macro. Esses autores propõem três tipos de mecanismos de relacionamento para analisar o nível micro: 
- Mecanismo Situacional - o ator individual é exposto a uma determinada situação social e esta situação o afeta de forma particular;

- Mecanismo de Ação-Formação - existe uma combinação específica de desejos e crenças individuais, as quais geram oportunidades e ações também específicas;

- Mecanismos Transformacionais - levam em conta a interação de certo número de indivíduos e o mecanismo, que difere dependendo da natureza da interação; apresentam como estas ações individuais são transformadas em alguma forma de resultado coletivo, intencional ou não.

Nas primeiras duas situações, os atores são individuais e o mecanismo é interno; na terceira, leva em conta que um grupo de atores e o mecanismo são tipicamente externos (ou social). Sob a ótica da gestão da cadeia de suprimentos, os mecanismos de socialização conduzem compradores e fornecedores a entender as peculiaridades e barreiras existentes no caminho para atingir eficiências operacionais (COUSINS et al., 2006). Os autores sugerem que os mecanismos de socialização, os quais são construídos a partir de valores comuns, do histórico e da longevidade da relação, são pré-requisitos importantes para os relacionamentos cooperativos e confiáveis de longo prazo. O quanto mais explícito e intensivo for o processo de socialização, mais rápido pode-se construir a confiança no relacionamento. As interações sociais entre compradores e vendedores estão, também, associadas à solução de problemas, reduções de custos e à troca de conhecimento de melhores práticas. Assim, os autores sugerem que quanto mais a socialização está presente no relacionamento interorganizacional, maiores são as possibilidades de se obter desempenho superior.

\subsection{Mecanismos formais de socialização}

Normas formais podem constituir um importante mecanismo de socialização e de formação do capital relacional e, por sua vez, podem facilitar ou restringir as ações dos atores (COLEMAN, 1988). Os mecanismos formais englobam as estruturas de controle de comportamento e resultados dos atores (STOUTHUYSEN; SLABBINCK; ROODHOOFT, 2012). Adicionalmente, a formalização está muito associada à padronização por meio de regras, procedimentos, normas e valores (KRAIMER, 1997), podendo ser consolidada de forma escrita ou não (CHOI; HONG, 2002).

Por um lado, como abordado por Van Maanen (1978), quanto mais formal for o processo, mais segregado e específico se tornam os papéis de cada ator. Por outro lado, os mecanismos formais de socialização aumentam a possibilidade de novos membros aceitarem a definição de situações oferecidas como significativas aos demais participantes dessas organizações ou redes de organizações. Isto aumenta o nível de compartilhamento de normas, valores e atitudes o que pode contribuir para um melhor direcionamento dos atores (CHOI; HONG, 2002). Os autores consideram que, por um lado, se o processo de formalização na cadeia de suprimentos refere-se ao nível que tal cadeia possui de controles por regras, procedimentos e normas que prescrevem os direitos e obrigações dos que dela participam, é importante reconhecer que tais regras existem no nível empresaempresa, ou mais precisamente no primeiro elo da cadeia (first tier) e não no nível sistêmico. Mais especificamente, não parece possível estabelecer ou impor uma formalização linear e simultânea a toda a cadeia de suprimentos. Por exemplo, o estudo desenvolvido por Martins et al. (2012) no setor automobilístico: os autores evidenciaram alinhamento entre comprador e fornecedor direto (first tier) de práticas consideradas valiosas para o cliente principal.

Em linha com o estudo de Cousins et al. (2006), o presente artigo sugere que os mecanismos formais podem impactar positivamente a geração de capital relacional, em que surge a primeira hipótese desta pesquisa:

H1: Os mecanismos formais de socialização impactam positivamente o relacionamento cliente-fornecedor.

\subsection{Mecanismos informais de socialização}

Os processos de socialização informais são similares às técnicas de "tentativa-e-erro", nas quais se aprende a partir da experiência (VAN MAANEN, 1978). Esse processo ocorre pela compreensão mútua de como compradores e vendedores podem trabalhar juntos, particularmente na transmissão, em suas organizações, dos objetivos e valores por interações informais e assim criar valor e congruência ou alinhamento entre suas ações (COUSINS et al., 2006; KRAIMER, 1997). Relacionamentos baseados em processos de socialização informal geralmente ultrapassam o ambiente físico dos negócios, direcionando maior enfoque aos aspectos informais e expressivos do relacionamento (COUSINS et al., 2006). As táticas de socialização informal podem aumentar o nível de confiança entre os membros de um relacionamento e oferecer maiores oportunidades, tempo e motivação para reforçá-lo.

O estudo de Ibarra (1993) leva em conta, por exemplo, o impacto do nível individual de análise e a informalidade da organização como uma forma de investigar as contribuições dos atributos individuais, formais e informais e a centralidade da rede para acessar o fluxo de informação e recursos necessários para geração de poder. Utiliza também os resultados 
comportamentais desse escopo. Ele concluiu que as fontes pessoais parecem possuir impacto significativo na inovação tecnológica e a autoridade formal está fortemente associada à inovação administrativa.

Neste contexto, a Teoria dos Jogos pode exercer certa contribuição. Sob esta ótica, interações futuras permitem aos atores que valorizem tais ações ou penalizem-se (HEIDE; MINER, 1992). Se um ator cooperar em determinado momento, o outro ator poderá reconhecer e recompensar o movimento cooperativo numa próxima oportunidade e viceversa. Sendo assim, se as recompensas futuras são importantes, a possibilidade de futura retaliação pode representar um risco e, consequentemente, um comportamento atual defensivo. Heide e Miner (1992) sugerem que as firmas, com o passar do tempo, conhecem-se melhor, porém adotam padrões estáveis de cooperação ou defensividade nos relacionamentos.

Complementando a abordagem da confiança, resolução conjunta de problemas e investimento em ativos específicos como gerador de vantagem competitiva, autores brasileiros, como Claro e Claro (2004), também postulam que coordenar relacionamentos colaborativos com fornecedores significa muito mais do que realizar uma boa compra. Os autores comprovaram os efeitos benéficos da relação interorganizacional ao apresentarem evidências de diferentes efeitos moderados na rede social. Assim sendo, apresenta-se a segunda hipótese deste artigo:

$\mathrm{H} 2$ : Os mecanismos informais de socialização impactam positivamente o relacionamento cliente-fornecedor.

\section{Capital relacional}

Capital relacional representa a habilidade das organizações em garantir benefícios a partir das redes de relacionamento (HUGHES; PERROW, 2011). Adicionalmente pode ser caracterizado como os recursos e conhecimentos pertencentes a uma estrutura social, os quais podem ser acessados ou mobilizados propositalmente (LIN; COOK; BURT, 2001). Por conta desta definição, a noção do capital relacional contém três ingredientes críticos: recursos pertencentes exclusivamente a uma estrutura social, acessibilidade a tais recursos pelos indivíduos e o uso desta mobilização de forma proposital. (COLEMAN, 1988).

Do ponto de vista de Lin (2001), se é assumido que o capital relacional captura os recursos valiosos em relacionamentos sociais, então a localização da rede pode facilitar, mas não necessariamente determinar, o acesso a melhores recursos. O autor reitera o capital relacional como algo mais que um relacionamento social, ele evoca os recursos próprios e acessados disponíveis e exclusivos a aquela rede social. Estes recursos não podem ser facilmente capturados sem identificar as características da rede e suas relações.
O conceito de capital relacional leva em conta que o grupo por si possui uma estrutura social e deve considerá-la como um todo e agregar as partes.

Entretanto, estabelecer relacionamentos não é uma questão rara ou difícil de imitar. $\mathrm{O}$ fato é que as vantagens competitivas de tais relacionamentos podem ser percebidas efetivamente como tais, se compradores e vendedores derem um passo à frente para os atributos e relacionamentos convencionais do mercado. A perspectiva do capital relacional favorece os atores de uma cadeia de suprimentos a tornarem-se mais engajados nas trocas sociais e tomar ações que, em outras situações, poderiam ser consideradas arriscadas (KIM et al., 2010). Neste contexto, Dyer e Singh (1998) propõem o conceito das rendas relacionais (relational rents), ou seja, lucro acima do normal gerado a partir da interação do relacionamento entre os atores, o qual não pode ser gerado por uma empresa isoladamente, sendo apenas possível se criado por meio de contribuições idiossincráticas de parcerias específicas. Tais rendas só são possíveis quando os parceiros, de comum acordo, combinam, trocam ou investem em ativos idiossincráticos, conhecimento e recursos que podem ser utilizados para minimizar custos de transação ou permitir a realização de ganhos pela combinação sinérgica desses ativos, conhecimentos ou recursos. Por conta disso e com base no estudo de Cousins et al. (2006), apresenta-se a terceira hipótese do presente estudo:

H3: A geração de capital relacional na cadeia de fornecimento está relacionada a ganhos de níveis superiores no relacionamento com fornecedores.

\section{Metodologia}

O método de pesquisa escolhido para este estudo foi o Survey (FREITAS et al., 2000; FORZA, 2002). A unidade de análise foi constituída por Executivos de Logística, Compras, Supply Chain ou áreas similares, colaboradores de empresas nacionais ou multinacionais em operação no Brasil desde, no mínimo, 2005. O objetivo de analisar a percepção dos executivos de Suprimentos se dá ao fato de que, de uma forma geral, o lado comprador é mais focado nos aspectos relacionais de construção de confiança e comprometimento e seus impactos no resultado. Isto porque, dentro de suas organizações, os compradores são medidos operacionalmente pela sua contribuição nos resultados da sua corporação e, desta forma, não é surpresa observar que eles estão mais preocupados com os possíveis caminhos que impactam diretamente seu desempenho (NYAGA; WHIPPLE; LYNCH, 2010). Fornecedores, apesar de interessarem-se também pelos aspectos relacionais, por outro lado, dedicam maior atenção à proteção de suas transações por meio dos contratos formais. 
O banco de dados utilizado nesta pesquisa foi estruturado a partir do banco de dados da CIESP e contatos estabelecidos pelos autores. Por se tratar de um banco de dados muito grande e por conta da indisponibilidade de recursos, uma fração do banco foi selecionada aleatoriamente. Uma validação prévia do banco de dados foi efetuada via telefone antes do início da pesquisa, com o objetivo de confirmar dados dos respondentes-alvo, como nome, cargo e e-mail, resultando em 273 contatos válidos.

O questionário utilizado foi inicialmente traduzido do original do estudo de Cousins et al. (2006), tomados os devidos cuidados sugeridos pela literatura para a tradução reversa e para garantir a integridade do instrumento de pesquisa (FORZA, 2002; DOUGLAS; CRAIG, 2007). Por conta de algumas limitações bibliográficas identificadas do estudo original, foi incluída uma escala (HAIR JUNIOR et al., 2005) ao questionário proposto por Cousins et al. (2006). Posteriormente, o questionário traduzido foi revisado e validado por quatro especialistas.

O pré-teste desta pesquisa foi encaminhado via $e$-mail para 20 pessoas, com retorno de 18 questionários (90\%). Estas pessoas foram escolhidas pela sua experiência na área de Compras e Supply Chain e, principalmente, por sua responsabilidade no desenvolvimento de negócios com fornecedores. $\mathrm{O}$ ambiente submetido foi semelhante ao ambiente utilizado para o questionário definitivo. Com o objetivo de avaliar o grau de confiabilidade de coerência interna, utilizou-se o Alfa de Crombach, obtendo-se resultados >0,65 como intensidade de associação, considerada como boa (HAIR JUNIOR et al., 2005).

$\mathrm{O}$ processo de refinamento do instrumento de pesquisa resultou em um questionário formado por 4 construtos e 13 variáveis observáveis, com uma variável a mais que o trabalho original de Cousins et al. (2006). O detalhamento da caracterização dos construtos é apresentado no Quadro 1.

As hipóteses foram testadas com a coleta de dados via Internet sob a plataforma Survey Monkey (www. surveymonkey.com). Estudos sugerem que pesquisas eletrônicas oferecem vantagens se comparadas ao correio convencional, por conta da velocidade, acessibilidade ao respondente, indicando um número inferior de não respondentes (BOYER et al., 2002), o que reforça a decisão tomada. A amostra foi composta por 91 respondentes, o que é considerado um resultado satisfatório, e esse resultado pode ser explicado pelo follow up realizado imediatamente após o envio dos questionários.

Os dados analisados no presente estudo são compostos por $42,9 \%$ empresas de pequeno porte, 16,5\% empresas de médio porte e 40,7\% empresas de grande porte. Para classificar as empresas segundo o tamanho, tomou-se por base os critérios adotados pelo SEBRAE - Serviço Brasileiro de Apoio às
Micro e Pequenas Empresas, que determina o porte da empresa conforme seu número de funcionários (www.sebrae.org.br).

Do ponto de vista do ramo de atuação da empresa respondente: $23,1 \%$ atua no setor de metal/mecânico; $16,5 \%$, na indústria metro-ferroviária; $15,4 \%$ são prestadores de serviços; $14,3 \%$ representam a indústria de base; $13,2 \%$ pertencem à indústria química/farmacêutica; $6,6 \%$ são da indústria automotiva/autopeças; $11 \%$ pertencem a outros setores (agroindústria, comercial, distribuidoras, importadoras, fabricação de máquinas e equipamentos). Quanto ao cargo/função dos respondentes: $28,6 \%$ são Compradores, Analistas ou Especialistas; 51,6\%, Gerentes, Supervisores ou Coordenadores; e 19,8\% são presidentes, diretores ou proprietários. Quanto ao grau de instrução: $11 \%$ possuem ensino superior incompleto; $26,4 \%$, superior completo; e $62,6 \%$ são pós-graduados.

\section{Análise dos resultados}

Depois da análise dos dados coletados, não foram identificados pontos fora da curva (outliers), tão pouco campos sem preenchimento (missing data). Em seguida, tomou-se por bem tratá-los com elevada amplitude, explorando máximo e mínimo e avaliar a assimetria dos dados. Notou-se que sob a ótica descritiva, os dados mostraram-se adequados.

Utilizou-se o modelo de equações estruturais construído a partir do software AMOS 7.0, utilizando-se a Estimação por Máxima Verossimilhança ou Maximum Likelihood (SCHUMACKER; LOMAX, 2004). O modelo detalhado resultante da saída do software Amos é apresentado na Figura 1. Nesta figura, são apresentados os construtos e seus respectivos itens de acordo com o Quadro 1, as relações conforme o embasamento teórico e as cargas resultantes dos testes estatísticos destas relações.

Adotou-se o alfa de Crombach para mensurar o índice de confiabilidade da consistência interna. Os resultados do teste indicaram valores satisfatórios associados aos construtos (HAIR JUNIOR et al., 2005). Foram encontradas as cargas de 0,83, 0,68 e 0,71 para Capital Relacional, Mecanismos Formais de Socialização e Mecanismos Informais de Socialização, respectivamente.

As medidas de ajuste do modelo levam em conta o número necessário estimado de coeficientes para alcançar determinado nível de ajuste. Os critérios adotados no presente trabalho estão resumidos no Quadro 2. Neste trabalho, usou-se o quiquadrado ajustado pelos graus de liberdade. Para este indicador, o limite inferior recomendado é de 1, enquanto o limite superior é 5 (SCHUMACKER; LOMAX, 2004).

Deve-se atentar que o quiquadrado, quando analisado sem relacioná-lo com os graus de liberdade de cada modelo, traz pouca contribuição. Sendo 


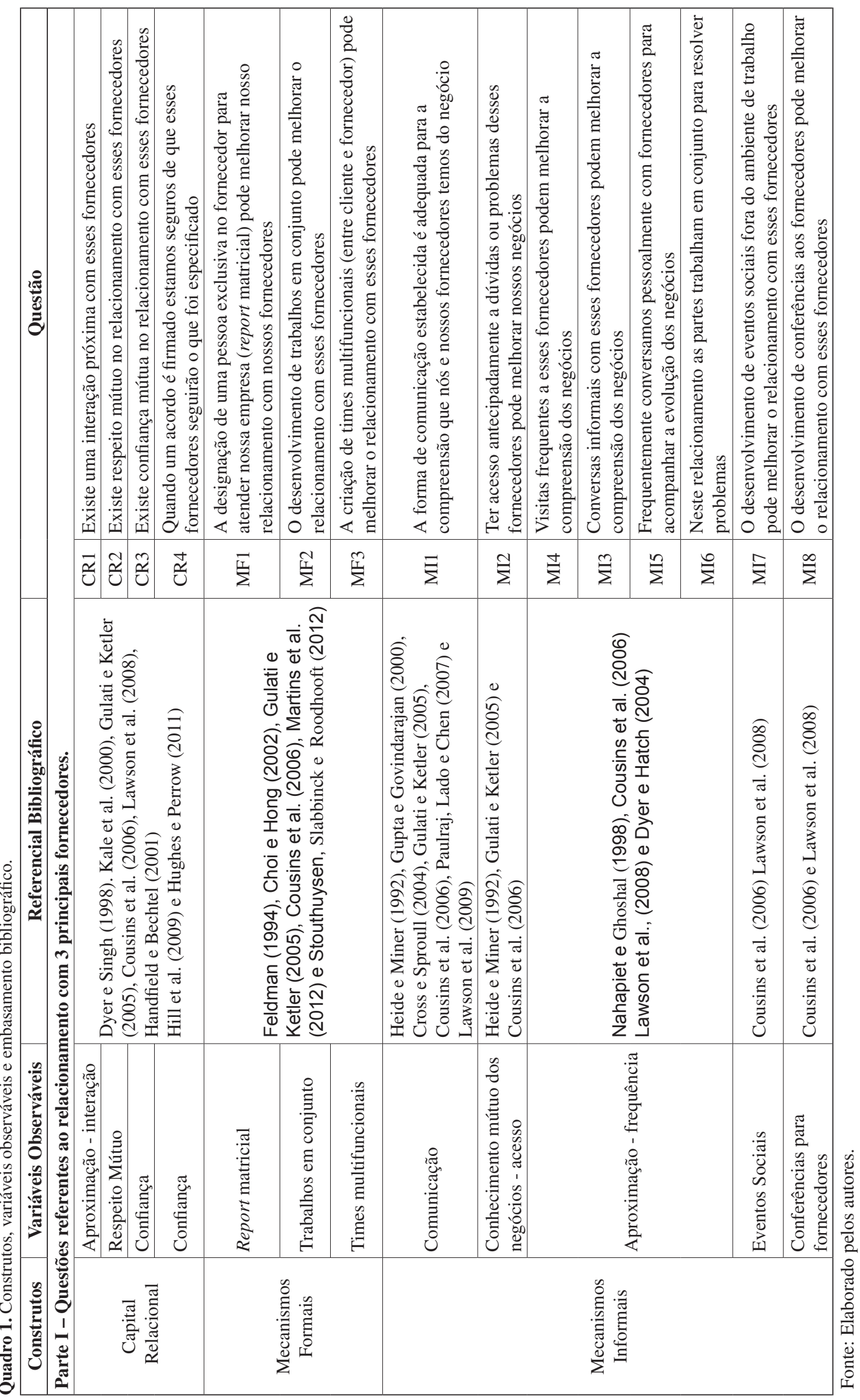




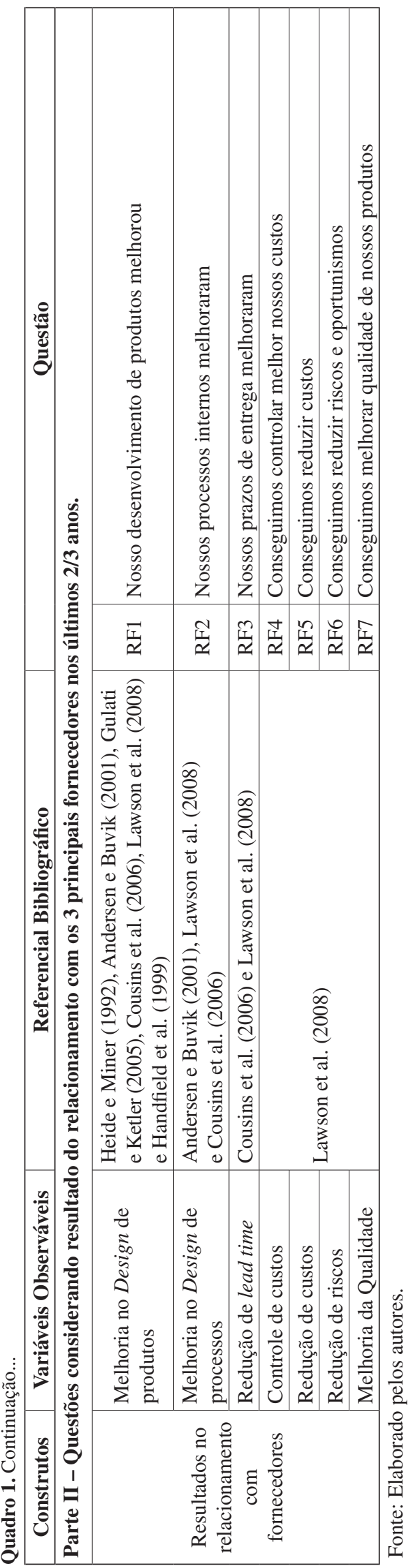




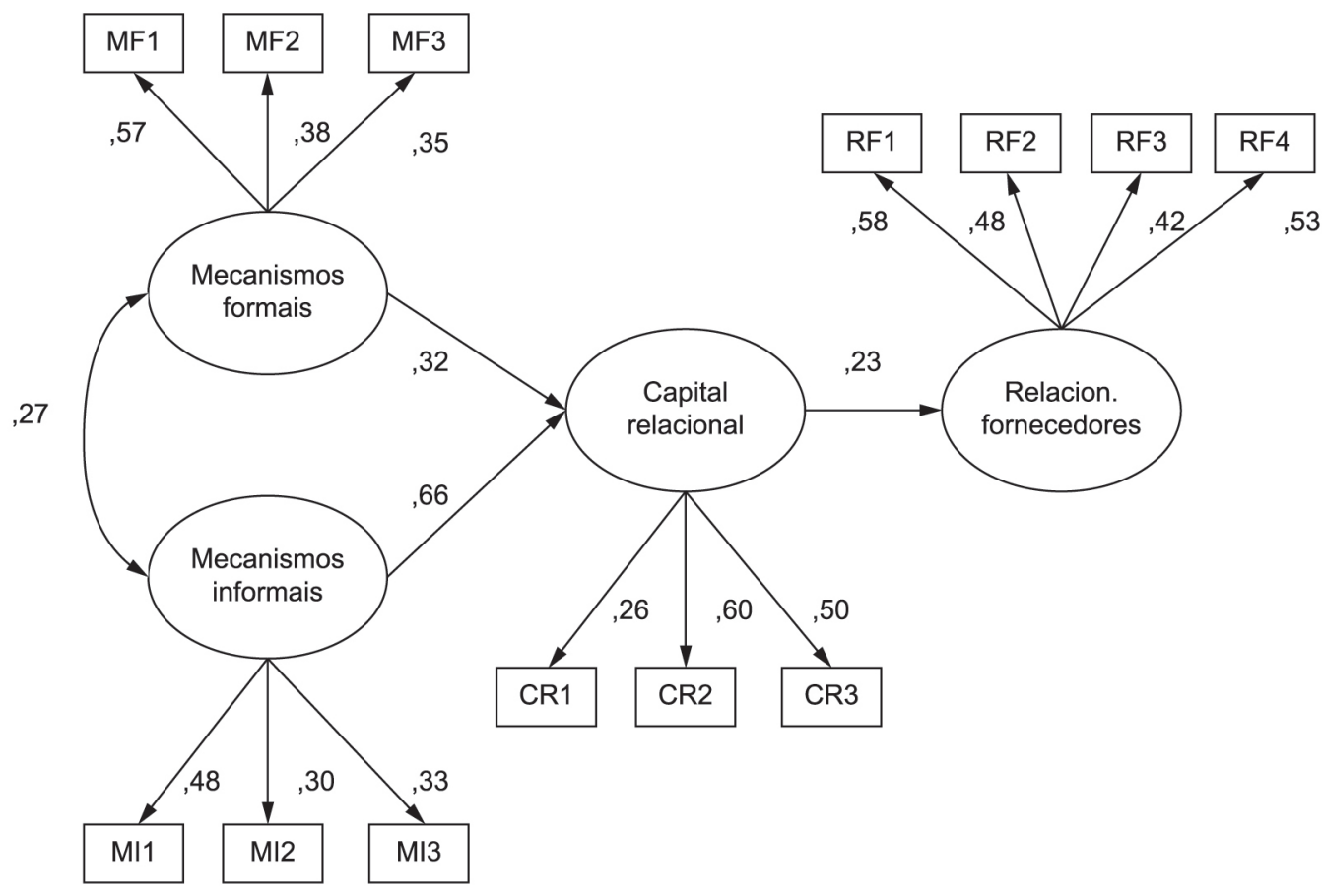

Figura 1. Modelo de Equações Estruturais. Fonte: Elaborado pelos autores. Nota: Estimativas padronizadas.

assim, buscou-se analisar o quiquadrado ajustado, cujo valor deve se situar entre 1 e 5 , a critério do nível de exigência do pesquisador. No presente estudo, o Quiquadrado encontrado foi 234,7 e os graus de liberdade, 57. Logo, o quiquadrado ajustado é de 4,1, considerado adequado para o estudo (SCHUMACKER; LOMAX, 2004).

Quanto ao índice absoluto GFI (ou Goodness to Fit Index), pode-se verificar que foi encontrado valor alto $(\mathrm{GFI}=0,859)$. Apesar de não existir um valor estabelecido para aceitação de modelos com base neste índice, quanto mais próximo de 1 , melhor o ajuste. O AGFI (Adjusted Goodness to Fit Index), de acordo com a literatura, deve assumir valores superiores a 0,8 , o que foi respeitado no modelo (valor encontrado AGFI=0,841). Portanto, considera-se, de maneira geral, como satisfatório o ajustamento do modelo pelos índices analisados (SCHUMACKER; LOMAX, 2004).

\section{Discussão e conclusões}

O presente estudo teve por objetivo analisar o impacto dos mecanismos de socialização na geração de capital relacional e observar se este impacto pode ser percebido como direcionador de resultados superiores no relacionamento com fornecedores. Para atender ao objetivo que o presente trabalho se propôs atingir, estabeleceram-se alguns objetivos específicos que nortearam o desenvolvimento desta pesquisa. O primeiro objetivo específico era compreender a conexão entre socialização e Capital relacional, revisando a literatura disponível e buscando o estado da arte em que o tema se encontra. Este objetivo foi atendido por meio da revisão bibliográfica.

As principais contribuições do estudo revelam a percepção de que as organizações que investem no estabelecimento específico de relações, como um ativo, empenhadas na troca de conhecimentos e recursos por mecanismos sociais informais e formais podem gerar, por meio dessas relações, ganhos acima do normal. Além disso, pelo processo de socialização, compradores e vendedores têm a oportunidade de reconhecer também os gaps que possam existir no caminho que eles estejam percorrendo durante o desenvolvimento de seus negócios, e assim redefinir a estratégia a ser tomada. E, finalmente, melhor definição dos mecanismos de socialização e seu impacto para acessar o capital relacional.

O objetivo seguinte era verificar a percepção dos executivos de Suprimentos no Brasil sobre o impacto dos mecanismos de socialização na geração de capital relacional e, consequentemente, na geração de resultados superiores. Este objetivo foi atendido pela pesquisa teórico-empírica desenvolvida no presente trabalho. Em resumo, os resultados mostram que os mecanismos de socialização informais possuem impacto significativo na geração de capital relacional $\mathrm{e}$, em contra partida, os mecanismos formais possuem impacto relativamente inferior.

Os resultados do estudo indicam que os mecanismos de socialização informais possuem influência direta, positiva e forte no relacionamento cliente-fornecedor, 
Quadro 2. Critérios adotados para ajuste do modelo e resultados obtidos.

\begin{tabular}{|c|c|c|c|}
\hline Tipo & Medidas & Nível de aceitação & Resultado obtido \\
\hline \multirow{6}{*}{$\begin{array}{l}\text { Medidas de Ajuste } \\
\text { Absoluto (por Máxima } \\
\text { Verossimilhança) }\end{array}$} & Quiquadrado $\left(\mathrm{X}^{2}\right)$ & não definido & 234,7 \\
\hline & Graus de liberdade & não definido & 57 \\
\hline & Número de parâmetros estimados & não definido & 34 \\
\hline & Nível de significância & A critério do pesquisador & $0,001 \checkmark$ \\
\hline & $\begin{array}{l}\text { Índice de Qualidade de ajuste } \\
\text { (GFI - Goodness to Fit) }\end{array}$ & 0 a 1 , sendo 1 o ajuste perfeito & $0,859 \checkmark$ \\
\hline & AGFI (Goodness to Fit ajustado) & 0 a 1 , sendo 1 o ajuste perfeito & $0,841 \checkmark$ \\
\hline \multirow{2}{*}{$\begin{array}{l}\text { Medidas de ajuste } \\
\text { Parcimonioso }\end{array}$} & Parsimonious fit index & 0 a 1 , sendo 1 o ajuste perfeito & $0,731 \checkmark$ \\
\hline & Quiquadrado $\left(\mathrm{X}^{2}\right)$ ajustado & 1 a 5 , sendo 1 o ajuste perfeito & $4,1 \checkmark$ \\
\hline
\end{tabular}

Fonte: Elaborado pelos autores a partir de Schumacker e Lomax (2004).

aceitando-se a segunda hipótese do presente estudo. Estes resultados também estão alinhados ao estudo de Cousins et al. (2006). Liker e Choi (2004) elucidam a necessidade de compreensão de como os fornecedores trabalham com a base para alcançar o desenvolvimento de negócios sustentáveis. Os autores sugerem ações simples como aprender sobre o negócio do fornecedor, respeitar as competências dos fornecedores e obter $\mathrm{o}$ comprometimento para o que eles denominam como "coprosperidade". Lawson, Tyler e Cousins (2008) perceberam que a integração e a aproximação de clientes e fornecedores está positivamente relacionada ao acúmulo de capital relacional. Eles sugerem que as empresas compradoras possuem condições suficientes de integrar certa quantidade de atividades com seus fornecedores-chave. Interações pessoais em diversos níveis das organizações, respeito e confiança mútua estão significativamente relacionadas a este contexto.

Os resultados indicam também que os mecanismos de socialização formais exercem influência positiva no relacionamento cliente-fornecedor, aceitando-se assim a primeira hipótese. Pode-se alinhavar os resultados obtidos nesta pesquisa com o trabalho de Cousins et al. (2006), sugerindo que as estruturas sociais não necessariamente conduzem ao capital relacional, mas podem facilitar os padrões informais dos comportamentos sociais necessários para a formação do capital relacional do grupo.

A terceira hipótese levava em conta verificar se o capital relacional impacta positivamente o resultado do relacionamento. A carga encontrada é positiva, porém muito baixa $(0,23)$, este resultado difere de achados anteriores em que esta relação, além de positiva, é muito mais forte (DYER; SINGH, 1998, CARR; PEARSON, 1999; ANDERSEN; BUVIK, 2001, HANDFIELD; BECHTEL, 2001; COUSINS et al., 2006; CAREY; LAWSON; KRAUSE, 2011). O resultado obtido sugere que não há evidências significativas para afirmar que o capital relacional tem influência sobre os ganhos de níveis superiores, pelo menos para esta amostra. Entretanto, esta percepção também é alvo de discussão na literatura há algum tempo. Dyer (1999) argumenta que a visão relacional por si só possui um conjunto independente e dinâmico de explanações para as conquistas de ganhos superiores oriundas de relacionamentos interorganizacionais. A percepção de Dyer (1999) neste contexto é de que selecionar qual proporção do sucesso pode ser atribuída à geração de capital relacional é um grande desafio.

Stouthuysen, Slabbinck e Roodhooft (2012) argumentam que a baixa significância do impacto dos mecanismos formais pode ser oriunda de uma possível complementariedade entre os mecanismos formais e informais. Mais precisamente, os autores sugerem que os mecanismos informais podem favorecer o desenvolvimento de mecanismos formais, enquanto que os mecanismos formais, se adotados isoladamente, podem criar conflitos irreversíveis entre os atores. Outros trabalhos levam em conta a necessidade de sincronização de expectativas entre clientes e fornecedores. Cox (2004) alerta para a existência intrínseca de conflito de interesses nesta relação e é tida como objetivo, a amenização destes conflitos. Souza, Moori e Marcondes (2005) evidenciaram que a qualidade é tida como fator de desempenho competitivo importante, tanto para clientes, como para fornecedores. Esta percepção também está em linha com Cox (2004), que elucida a funcionalidade de serviços/bens como um fator determinante de manipulação e possível instrumento de sincronização entre clientes e fornecedores.

Além dos fatores anteriormente citados, outras barreiras à percepção de ganhos no relacionamento também podem ser consideradas relevantes neste contexto. Dyer e Hatch (2004) sinalizam a rigidez dos processos internos como uma barreira, pois a possibilidade de abrir e transferir informações confidenciais aos fornecedores é percebida como difícil e possui uma série de barreiras internas. Seu estudo leva em conta que diversos executivos temem não valer a pena correr o risco de compartilhar este tipo de informação. 
Outra razão para o resultado obtido quanto ao impacto do capital relacional ser de baixa intensidade pode se dar ao fato do crítico momento econômico em que o País se encontrava quando da realização da presente pesquisa. Tal ambiente ainda apresentava um cenário incerto e complexo com quedas de faturamento significativas, demissões de funcionários, sinalizando um desaceleramento global. Este ponto deve ser levado em consideração, como fundamenta Cousins e Lawson (2006): os autores destacaram como crítica a percepção dos gerentes de compras entrevistados, pois eles percebem que intuitivamente a socialização possui um impacto significativo no desempenho, porém, por conta da dificuldade de se mensurar e justificar estas ações, a socialização passa a ser a primeira coisa a ser desconsiderada em momentos de crise, o que parece um contrassenso. Assim sendo, tais argumentações justificam teoricamente a percepção de impacto baixo da geração de capital relacional no relacionamento entre clientes e fornecedores.

\section{Contribuições, limitações e direcionamento futuro}

Apesar de todo o cuidado no desenvolvimento, este estudo não está isento de algumas limitações. A primeira limitação leva em conta a escolha da amostra utilizada. Apesar do possível viés por conta de ser oriunda de um banco de dados desenvolvido pelos autores, a amostra mostrou-se estável e foi construída com muito cuidado e critério. Entretanto, leva-se em consideração a possibilidade de não retratar, em sua amplitude, a população-alvo. Outra limitação é quanto ao número de respondentes $(\mathrm{n}=91)$. Isto impactou diretamente uma boa mensuração do modelo adotado. Uma amostra maior poderia apresentar maior sustentação aos dados analisados. Neste contexto, uma nova oportunidade de estudos emerge baseada em maior amplitude da amostra. Ressalta-se que uma limitação que pode ter impacto no presente trabalho é quanto ao ambiente econômico em que o País se encontrava no momento da pesquisa. Uma alternativa a este ponto é desenvolver uma nova pesquisa no momento em que a economia demonstrar maior estabilidade.

Uma sugestão de pesquisa futura seria a contribuição que uma análise de amostra estratificada poderia oferecer para a identificação de variações por conta do porte da empresa, número de funcionários, faturamento, escolaridade dos executivos, estrutura de mercado e ramo de atividade. Além disso, uma oportunidade de pesquisa que surge à luz do presente estudo leva em conta a possível refutação dada à percepção de ganho no relacionamento cliente-fornecedor. Cabe um convite à exploração dos motivos intrínsecos e a busca não necessariamente de um desenho ideal, porém de um desenho de relacionamento mais equilibrado.
Durante a investigação teórica acerca do presente estudo, emergiram alguns trabalhos de sociologia econômica (MARTES et al., 2006), que, possivelmente, poderiam contribuir positivamente nesta direção, quiçá aprofundando os conhecimentos nessa linha de estudos. Sugere-se, também, o desenvolvimento de um estudo longitudinal para compreender o processo de evolução dos mecanismos de socialização no desempenho, assim como um estudo qualitativo dos temas aqui abordados, o que poderiam ser de significativa contribuição para a interpretação dos dados.

Finalmente, este artigo oferece algumas contribuições do ponto de vista teórico:

a) Melhor esclarecimento dos mecanismos de socialização interorganizacionais e seu impacto na geração de valor nos relacionamentos entre clientes e fornecedores, por meio do desenvolvimento teórico-empírico;

b) Refinamento e aprimoramento do instrumento de pesquisa utilizado;

c) Identificação, operacionalização e análise dos principais conceitos de capital relacional, mecanismos de socialização e desempenho.

Do ponto de vista metodológico, a amostra pesquisada mostrou-se adequada e os resultados coesos. Logo, entende-se que as conclusões obtidas podem refletir a população-alvo que este projeto propôs avaliar. Além disso, o estudo oferece suporte a melhores práticas corporativas, nas quais gerentes e dirigentes poderão reavaliar a criação e desenvolvimento de seus relacionamentos com fornecedores.

\section{Referências}

ANDERSEN, O.; BUVIK, A. Inter-firm co-ordination: international versus domestical buyer-seller relationships. Omega, v. 29, n. 2, p. 207-219, 2001. http://dx.doi. org/10.1016/S0305-0483(00)00043-8

BOURDIER, P. Le Capital Social. Actes de la recherche en sciences sociales, v. 31, p. 2-3, 1980.

BOYER, K. K. et al. Print versus electronic surveys: a comparison of two data collection methodologies. Journal of Operations Management, v. 20, n. 4, p. 357-373, 2002. http://dx.doi.org/10.1016/ S0272-6963(02)00004-9

CAREY, S.; LAWSON, B.; KRAUSE, D. R. Social Capital Configuration, Legal Bonds And Performance n Buyer-Supplier Relationships. Journal of Operations Management, v. 29, n. 4, p. 277-288, 2011. http:// dx.doi.org/10.1016/j.jom.2010.08.003

CARR, A. S.; PEARSON, J. N. Strategically managed buyer-supplier relationships and performance outcomes. Journal of Operations Management, v. 17, n. 5, p. 497-519, 1999. http://dx.doi.org/10.1016/ S0272-6963(99)00007-8 
CAMPBELL, A. Buyer-supplier partnerships: flip sides of the same coin? Journal of Business \& industrial Marketing, v. 12, n. 6, p. 417-434, 1997. http://dx.doi. org/10.1108/08858629710190295

CLARO, D. P.; CLARO, P. B. O. Gerenciando relacionamentos colaborativos com fornecedores. RAE Revista de Administração de Empresas, v. 44, n. 4, p. 68-79, 2004. http://dx.doi.org/10.1590/ S0034-75902004000400003

CHOI, T. Y.; HONG, Y. Unveiling the Structure of Supply Networks: case studies in Honda, Acura and Daimler Chrysler. Journal of Operations Management, v. 20, n. 5, p. 469-493, 2002. http://dx.doi.org/10.1016/ S0272-6963(02)00025-6

COLEMAN, J. S. Social Capital in the Creation of Human Capital. The American Journal of Sociology, v. 94, p. 95-120, 1988. http://dx.doi.org/10.1086/228943

COUSINS, P. D. et al. Creating supply chain relational capital: The impact of formal and informal socialization processes. Journal of Operations Management, v. 24, n. 6, p. 851-863, 2006. http://dx.doi.org/10.1016/j. jom.2005.08.007

COUSINS, P. D.; LAWSON, B. What you measure is not necessarily what you get: The role of socialization in managing supplier relationships. Supply Chain Management Research Group: Manchester Business School - Executive Briefing, 2006.

COUSINS, P. D.; MENGUC, B. The Implications of Socialization and Integration in Supply Chain Management. Journal of Operations Management, v. 24, n. 5, p. 604-620, 2006. http://dx.doi.org/10.1016/j. jom.2005.09.001

CROSS, R.; SPROULL, L. More than ans Answer: Information Relatuionships for Actionable Knowledge. Organization Science, v. 15, n. 4, p. 446-462, 2004. http://dx.doi.org/10.1287/orsc.1040.0075

COX, A. Business Relationship Alignment: on the commensurability of value capture and mutuality in buyer and supplier exchange. Supply Chain Management: An International Journal, v. 9, n. 5 p. 410-420, 2004. http://dx.doi.org/10.1108/13598540410560793

DYER, J.; SINGH, H. Relational View: Cooperative Strategy and sources of interorganizational competitive advantage. Academy of Management Review, v. 23, n. 4, p. 660-679, 1998.

DYER, J. Response to relational view commentary. The Academy Management Review, v. 24, n. 2, p. 185-186, 1999.

DYER, J.; HATCH, N. W. Using Supplier Networks to Learn Faster. MIT Sloan Management Journal, p. 57-63, 2004.

DOUGLAS, S.; CRAIG, C. S. Collaborative and Iterative Translation: An Alternative Approach to Back Translation. Journal of International Marketing, v. 15, n. 1, p. 30-43, 2007. http://dx.doi.org/10.1509/jimk.15.1.030

FELDMAN, D. Who's Socializing whom? The impact of socializing newcomers on insiders, work groups and organization. Human Resource Management
Review, v. 4, n. 3, p 213-233, 1994. http://dx.doi. org/10.1016/1053-4822(94)90013-2

FLYNN, B. B.; HUO, B.; ZHAO, X. The impact of supply chain integration on performance: A contingency and configuration approach. Journal of Operations Management, v. 28, p. 58-71, 2010. http://dx.doi. org/10.1016/j.jom.2009.06.001

FORZA, C. Survey research in operations management: a process-based perspective. International Journal of Operations and Production Management, v. 22, n. 2, p. 152-194, 2002. http://dx.doi. org/10.1108/01443570210414310

FREITAS, H. et al. O Método de Pesquisa Survey. Revista de Administração RAUSP, v. 35, n. 3, p. 105-112, 2000.

GRANOVETTER, M., Ação Econômica e Estrutura Social: O Problema da Imersão. RAE-Eletrônica, v. 6, n. 1, 2007. http://dx.doi.org/10.1590/S1676-56482007000100006

GULATI, R. Alliances and Network. Strategic Management Journal, v. 19, n. 4, p. 293-317, 1998. http://dx.doi. org/10.1002/(SICI)1097-0266(199804)19:4<293::AIDSMJ982>3.0.CO;2-M

GUlATI, R.; GARGiUlo, M, Where Does Interorganizational Networks Come From? American Journal of Sociology, v. 104, n. 5, p. 1439-1493, 1999. http://dx.doi.org/10.1086/210179

GULATI, R.; KLETTER, D. Shrinking Core, Expanding Periphery: The Relational Architeture of High Performing Organizations. California Management Review, v. 47, n. 3, p. 77-104, 2005. http://dx.doi.org/10.2307/41166307

GUPTA, A. K.; GOVINDARAJAN, V. Knowledge flows within multinational corporations. Strategic Management Journal, v. 21, n. 4, p. 473-496, 2000. http://dx.doi.org/10.1002/(SICI)10970266(200004)21:4<473::AID-SMJ84>3.0.CO;2-I

HANDFIELD, R. B. et al. Involving suppliers in new products development. California Management Review, v. 42, n. 1, p. 59-82, 1999. http://dx.doi. org/10.2307/41166019

HANDFIELD, R. B.; BECHTEL, C. The Role of Trust and Relationship Structure in Improving Supply Chain Responsiveness. Industrial Marketing Management, v. 31, n. 4, p. 367-382, 2001. http://dx.doi.org/10.1016/ S0019-8501(01)00169-9

HAIR JUNIOR, J. F. et al. Fundamentos de Métodos de Pesquisa em Administração. São Paulo: Editora Bookman, 2005. 471 p.

HAKANSSON, H.; FORD, D. How should companies interact in business networks? Journal of Business Research, v. 55, p. 133-139, 2002. http://dx.doi. org/10.1016/S0148-2963(00)00148-X

HEDSTRÖM, P.; SWEDBERG, R. Social Mechanisms, An Analytical Approach to Social Theory. Cambridge: Cambridge University Press, 1998. http://dx.doi. org/10.1017/CBO9780511663901

HEDSTRÖM, P. Dissection the Social: On the Principals of Analytical Sociology. Cambridge: Cambridge University Press, 2005. 177 p. http://dx.doi.org/10.1017/ CBO9780511488801 
HEIDE, J. B.; MINER, A. S. The Shadow of the future: effects of Antecipated iunteraction and frequency of contact on buyer-seller cooperation. Academy of Management Journal, v. 35, n. 2, p. 265-291, 1992. http://dx.doi.org/10.2307/256374

HILL, J. A. et al. The effect of unethical behavior on trust in a buyer-supplier relationship: The mediating role of psychological contract violation. Journal of Operations Management, v. 27, n. 4, p. 281-293, 2009. http:// dx.doi.org/10.1016/j.jom.2008.10.002

HUGHES, M.; PERRONS, R. K. Shaping and re-shaping social capital in buyer-supplier relationships. Journal of Business Research, v. 64, p. 164-171, 2011. http:// dx.doi.org/10.1016/j.jbusres.2009.12.009

IBARRA, H. Network Centrality, Power and Innovation Involvement: Determinants of Technical and Administrative Roles. Academy of Management Review, v. 36, n. 3, p. 471-501, 1993. http://dx.doi. org/10.2307/256589

KALE, P.; SINGH, H.; PERLMUTTER, H. Learning and protection of Proprietary assets in strategic alliances: building relational capital. Strategic Management Journal, v. 21, n. 3, p. 217-237, 2000. http://dx.doi. org/10.1002/(SICI)1097-0266(200003)21:3<217::AIDSMJ95>3.0.CO;2-Y

KIM, K. K. et al. Inter-organizational cooperation in buyer-supplier relationships: Both perspectives. Journal of Business Research, v. 63, n. 8, p. 863-869, 2010. http://dx.doi.org/10.1016/j.jbusres.2009.04.028

KRAIMER, M. L. Organizational Goals and Values: A socialization model. Human Resource Management Review, v. 7, n. 4, p. 425-447, 1997. http://dx.doi. org/10.1016/S1053-4822(97)90028-0

LAWSON, B., TYLER, B. B.; COUSINS, P. D. Antecedents and Consequences of Social Capital on Buyer Performance Improvement. Journal of Operations Management, v. 26, n. 3, p. 446-460, 2008. http:// dx.doi.org/10.1016/j.jom.2007.10.001

LAWSON, B. et al. Knowledge sharing in interorganization product development teams: the effect of formal and informal socialization mechanisms. The Journal of Product Innovation Management, v. 26, p. 156-172, 2009. http://dx.doi. org/10.1111/j.1540-5885.2009.00343.x

LIN, N.; COOK, K; BURT, R. S. Social Capital: Theory and Research. 1. ed. New York: Aldine de Gruyter, 2001. http://dx.doi.org/10.1017/CBO9780511815447

LIN, N. Social Capital: A Theory of Social Structure and Action. 1. ed. Cambridge: Cambridge University Press, 2001. http://dx.doi.org/10.1017/CBO9780511815447

LOUIS, M. R. Surprise and Sense Making: What Newcomers Experience in Entering Unfamiliar Organizational Settings. Administrative Science Quaterly, v. 25, n. 2, p. 226-251, 1980. http://dx.doi.org/10.2307/2392453

LIKER, J. K.; CHOI, T.Y. Building Deep Supplier Relationships. Harvard Business Review, v. 82, n. 12, p. 104-113, dec. 2004

LUMINEAU, F.; HENDERSON, J. E. The influence of relational experience and contractual governance on the negotiation strategy in buyer-supplier disputes. Journal of Operations Management, v. 30, n. 5, p. 382-395, 2012. http://dx.doi.org/10.1016/j.jom.2012.03.005

MARTES, A. C. B. et al. Forum - Redes Sociais e Interorganizacionais. RAE Revista de Administração de Empresas, v. 46, n. 3, p. 10-15, 2006. http://dx.doi. org/10.1590/S0034-75902006000300002

MARTINS, R. S. et al. Strategic Alignment in the Brazilian Automotive Chain. Journal of Operations and Supply Chain Management, v. 5, n. 1, 2013.

NAHAPIET, J.; GHOSHAL, S. Social Capital, Intellectual Capital and the Organizational Advantage. Academy of Management Review, v. 23, n. 2, p. 242-268, 1998.

NYAGA, G. N.; WHIPPLE, J.; LYNCH, D. Examining supply chain relationships: Do buyer and supplier perspectives on collaborative relationships differ? Journal of Operations Management, v. 28, p. 101-114, 2010. http://dx.doi.org/10.1016/j.jom.2009.07.005

OLIVER, C. Determinantes of Interorganizational Relationships: Integration and Future Directions. Academy of Management Review, v. 15, n. 2, p. 241-265, 1990.

PAULRAJ, A.; LADO, A. A.; CHEN, I. J. Inter-organizational communication as a relational competency: antecedents and performance outcomes in collaborative buyer-supplier relationships. Journal of Operations Management, v. 26, n. 1, p. 45-64, 2008. http://dx.doi.org/10.1016/j. jom.2007.04.001

SACOMANO NETO, M.; PIRES, S. R. I. Medição de desempenho em cadeias de suprimentos: um estudo na indústria automobilística. Gestão \& Produção, v. 19, n. 4, p. 733-746, 2012. http://dx.doi.org/10.1590/ S0104-530X2012000400006

SCHOENHERR, T. et al. Research opportunities in purchasing and supply management. International Journal of Production Research, v. 50, n. 6, p. 45564579, 2012. http://dx.doi.org/10.1080/00207543.2011 .613870

SCHUMACKER, R. E.; LOMAX, R. G. A Beginner's Guide to Structural Equation Modeling. 2. ed. New Jersey: Lawrence Elbaum Associates Publishers, 2004. SKJOETT-LARSEN, T. Supply Chain Management: a new challenge for researchers and managers in Logistics. International Journal of Logistics Management, v. 10, n. 2, p. 41-53, 1999. http://dx.doi. org/10.1108/09574099910805987

SOUZA, M. F. S.; MOORI, R. G.; MARCONDES, R. C. Sincronismo entre clientes e fornecedores. RAE: Revista de Administração de Empresas, v. 45, n. 4, p. 36-49, 2005.

STOUTHUYSEN, K.; SLABBINCK, H. ROODHOOFT, F. Controls, service type and perceived supplier performance in interfirm service exchanges. Journal of Operations Management, v. 30, n. 5, p. 423-435, 2012. http:// dx.doi.org/10.1016/j.jom.2012.01.002

TERPEND, R. et al. Buyer Supplier Relationships: Derived Value over Two Decades. Journal of Supply Chain Management, v. 44, n. 2, p. 28-55, 2008. http://dx.doi. org/10.1111/j.1745-493X.2008.00053.x 
WANG, E.; TAI, J.; VARUN, G. Examining the Relational Benefits of Improved Interfirm Information Processing Capability in Buyer-Supplier Dyads. MIS Quarterly, v. 37, n. 1, p. 149-173, 2013.

VILLENA, V. H.; REVILLA, E.; CHOI, T. Y. The dark side of buyer-supplier relationships: A social capital perspective. Journal of Operations Management, v. 29, n. 6, p. 561-576, 2011. http://dx.doi.org/10.1016/j. jom.2010.09.001
VAN MAANEN, J. People Processing: Strategies of Organization Socialization. Organization Dynamics, v. 1, p. 19-36, 1978. http://dx.doi. org/10.1016/0090-2616(78)90032-3

VASCONCELOS, M. C. R. L.; MILAGRES, R.; NASCIMENTO, E. Estratégia de Relacionamento entre os membros da Cadeia Produtiva no Brasil: Reflexões sobre o Tema. Gestão \& Produção, v. 12, n. 3, p. 393-404, 2005. http://dx.doi.org/10.1590/ S0104-530X2005000300009 